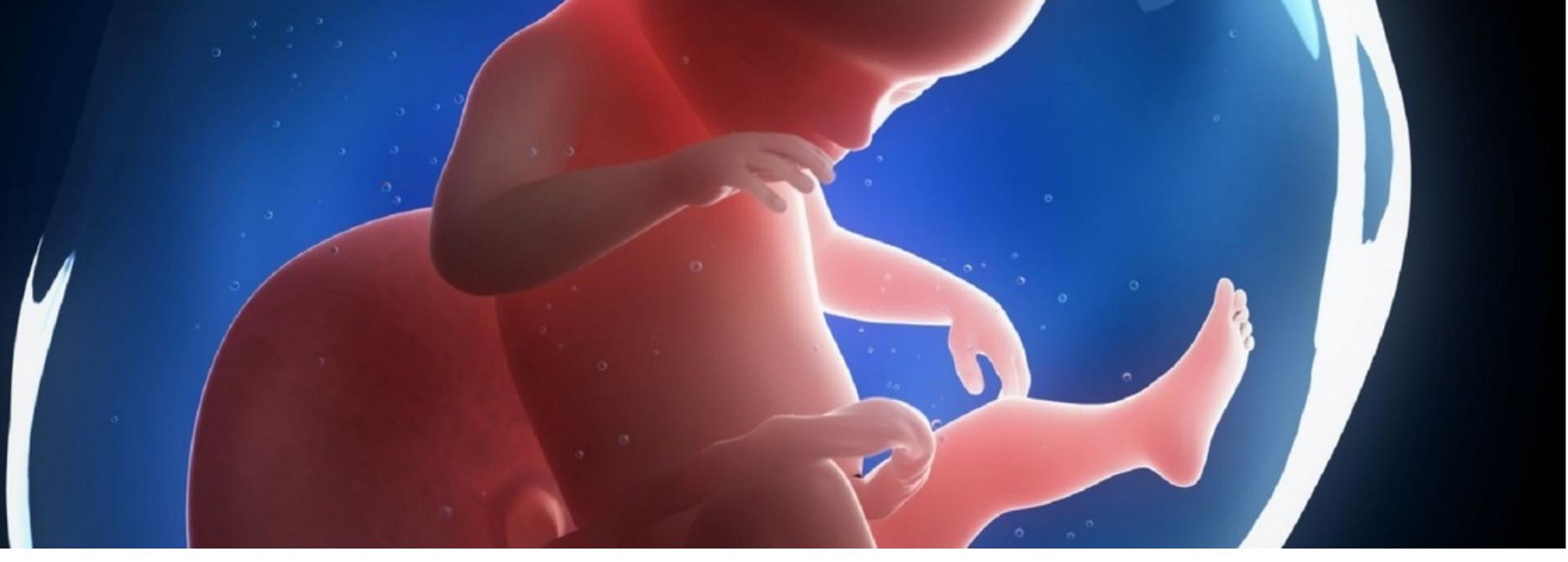

PREVALENCE ALTERATION OF DOPPLER UTERINE ARTERIES BETWEEN WEEK 20 - 24 OF GESTATION IN LOW RISK POPULATION

Authors: $\quad$ Linda Patricia L. Meléndez Pérez, Monica Andrea Beltran Avendaño

Submitted: $\quad$ 6. March 2018

Published: $\quad$ 9. March 2018

Volume: $\quad 5$

Issue: 2

Affiliation: Universidad Industrial de Santander

Languages: Spanish, Castilian

Keywords: $\quad$ Doppler uterine artery, low risk population, second trimester

DOI: $\quad$ 10.17160/josha.5.2.394

JOSHA

josha.org
Journal of Science,

Humanities and Arts

JOSHA is a service that helps scholars, researchers, and students discover, use, and build upon a wide range of content 


\title{
PREVALENCE ALTERATION OF DOPPLER UTERINE ARTERIES BETWEEN WEEK 20 - 24 OF GESTATION IN LOW RISK POPULATION
}

\author{
PREVALENCIA DE LA ALTERACIÓN DEL DOPPLER DE ARTERIAS \\ UTERINAS ENTRE SEMANA 20 - 24 EN PACIENTES DE BAJO RIESGO
}

\begin{abstract}
Linda Patricia Meléndez Pérez* Monica Andrea Beltran Avendaño**.

* Medica Residente de Ginecología y Obstetricia de tercer año, Departamento de Ginecologia y Obstetricia, Universidad Industrial de Santander (UIS), Bucaramanga, Colombia. Escuela de Medicina. Dirección de correspondencia: Autopista Bucaramanga - Floridablanca No. 149 - 164 Torre 2 Apto 1001, correo electronico: $\underline{\text { inda2158019@ @ correo.uis.edu.co. }}$

** Especialista en Medicina Materno Fetal, Docente de catedra del Departamento de Ginecología y Obstetricia, Universidad Industrial de Santander, Bucaramanga, Colombia.
\end{abstract}

\begin{abstract}
:
Background: In Colombia, patients classified as obstetric low risk have maternal or fetal adverse outcomes despite the process of classification of prenatal controls, within these outcomes are pathologies associated with the alteration in placentation. Doppler of uterine arteries (DUA) is a useful method to predict complications derived from inadequate placentation, for this reason we decided to evaluate this diagnostic tool. In Bucaramanga and its metropolitan area (AMB), the prevalence alteration of DUA between week 20 and 24 of gestation in low risk obstetric patients is not known, for this reason the current study was designed.
\end{abstract}

Objective: Determine the prevalence alteration of Doppler uterine artery (DUA) and its parameters (pulsatility index (PI) and resistance (RI) and Notch) in low risk obstetric patients between week 20 - 24.6 of gestation in Bucaramanga and its metropolitan area.

Settings: Patients were recruited in basic hospital care centers of AMB and the DUA was performed in the Hospital Universitario de Santander (HUS)

Study design: Prospective cross-sectional study, in which low-risk obstetric patients with 
less than 24.6 weeks of gestation were recruited. The obstetric risk was corroborated and fetal biometry and DUA were performed between week 20 - 24.6 to perform fetal biometry and DUA. The statistical analysis of the data was performed with the Stata program 12.1. The ethical considerations were approved by the medical ethics committee of the UIS.

Results: 120 patients were recruited between 18 and 35 years old and $49.2 \%$ (59) were primigravid. The median gestational age at the time of the DUA was 21.6 weeks. There was an abnormality IP in $15.0 \%$ (18) of the patients (95\% CI from $9.1 \%$ to $22.7 \%$ ), of the IR in $25.0 \%$ (30) (95\% CI from $17.3 \%$ to $33.7 \%$ ) and presence of Notch in $10.8 \%$ (13) (95\% CI from $5.9 \%$ to $17.8 \%)$. Of $18(15.0 \%)$ patients who had impaired PI 2 had normal RI and the Notch was absent in 6. The three parameters were altered in $11(9.2 \%)$ patients.

Conclusions: The IP alteration was $15.0 \%$ (18), which was expected according the selection criteria we used to define obstetric low risk population. Taking into account the number of births / year that occur in Bucaramanga and its metropolitan area, this prevalence is not negligible and we consider that is necessary to continue this line of research to establish the relevance of performing the DUA in the low-risk population in the second trimester.

Keywords: Doppler uterine artery, low risk population, second trimester

Palabras claves: Doppler de arterias uterinas, bajo riesgo obstétrico, segundo trimestre.

\section{RESUMEN:}

Antecedentes: En Colombia las pacientes clasificadas como bajo riesgo obstétrico presentan desenlaces maternos o fetales adversos a pesar del proceso de clasificación de los controles prenatales, dentro de estos desenlaces se encuentran patologías asociadas a la alteración en la placentación. El Doppler de arterias uterinas (DUA) es un método útil para predecir complicaciones derivadas de una inadecuada placentación, razón por la cual se inicia una línea de investigación para evaluarlo. El propósito de este trabajo fue evaluar en Bucaramanga y su área metropolitana $(\mathrm{AMB})$ la prevalencia de alteración del DUA en pacientes de bajo riesgo obstétrico entre la semana 20 - 24,6 de gestación, debido a la ausencia de esta información en la región.

Objetivo: Determinar la prevalencia de alteración del Doppler de arterias uterinas (DUA) y sus parámetros (Índice de pulsatilidad (IP) y resistencia (IR) y Notch) en pacientes de bajo 
riesgo obstétrico entre la semana 20 - 24,6 de gestación en Bucaramanga y su área metropolitana.

Instituciones: Las pacientes fueron reclutadas en centros de atención hospitalaria del primer nivel de AMB y el DUA fue realizado en el Hospital Universitario de Santander (HUS)

Diseño del estudio: Estudio transversal prospectivo, en el que se reclutaron pacientes de bajo riesgo obstétrico con menos de 24,6 semanas de gestación, se corroboró mediante interrogatorio su riesgo obstétrico y se citó entre la semana 20 - 24,6 para realizar biometría fetal y DUA. El análisis estadístico de los datos se realizó con el programa Stata 12,1. Las consideraciones éticas fueron aprobadas por el comité de ética médica de la UIS.

Resultados: Se reclutaron 120 pacientes entre los 18 y 35 años y el 49,2\% (59) eran primigestantes. La mediana de edad gestacional al momento del DUA fue de 21,6 sem. Hubo anormalidad del IP en el 15,0\% (18) de las pacientes (IC 95\% de 9.1\% a 22.7\%), del IR en el $25,0 \%$ (30) (IC 95\% de $17.3 \%$ a 33.7\%) y presencia del Notch en el 10,8\% (13) (IC 95\% de $5.9 \%$ a $17.8 \%)$. De $18(15,0 \%)$ pacientes que tenían IP alterado 2 tenían IR normal y el Notch se encontraba ausente en 6. Los 3 parámetros estaban alterados en el $11(9,2 \%)$ pacientes.

Conclusiones: La alteración del IP fue del 15,0\% (18) lo cual era esperado teniendo en cuenta los criterios de selección que utilizamos para definir población de bajo riesgo obstétrico. Teniendo en cuenta la cantidad de nacimientos/año que se presentan en Bucaramanga y su área metropolitana esta prevalencia no es despreciable y se considera necesario continuar con esta línea de investigación para establecer la pertinencia de realizar el DUA en población de bajo riesgo en $2^{\circ}$ trimestre.

\section{INTRODUCCIÓN}

En Colombia se ha desarrollado la estrategia de clasificación de riesgo obstétrico de las gestantes en dos categorías según las guías del Ministerio de la Protección Social (MINPRO): alto y bajo riesgo obstétrico. Se considera alto riesgo obstétrico aquellas pacientes con: 1 factor de riesgo alto para trastornos hipertensivos asociados al embarazo o presencia de dos o más factores de riesgo moderados para esta condición, pacientes con puntuación $\geq 3$ en la escala de Herrera y Hurtado o alto riesgo para trabajo de parto pre - término por presencia factores de riesgo reproductivos y ginecológicos $(1,2)$. Las pacientes que no cumplen con 
los anteriores criterios son consideradas de bajo riesgo obstétrico; sin embargo, en la práctica clínica se observan desenlaces maternos o fetales adversos en esta población $(3,4)$. En las poblaciones de bajo riesgo obstétrico se han visto desenlaces adversos como: preclampsia, restricción del crecimiento intrauterino, feto pequeño para la edad gestacional (FPEG), parto pretérmino < de 33 semanas, muerte fetal intrauterina y desprendimiento de placenta; todas estas, condiciones asociadas a la alteración en la invasión del trofoblasto $(3,4,5,6)$. En la literatura se ha reportado como el Doppler de arterias uterinas puede identificar la alteración en el proceso de placentación, mostrando desde una disminución del flujo sanguíneo en diástole hasta flujo reverso en los casos más severos $(5,7)$. De allí surge la posibilidad de anticipar los trastornos derivados de la placentación anormal por medio del Doppler de arterias uterinas en pacientes clasificadas como bajo riesgo, debido a que estas noxas tienen alta incidencia en la población gestante y generan un impacto social relevante.

Dado que se desconoce la prevalencia de las alteraciones del Doppler de Arterias Uterinas en pacientes clasificadas como bajo riesgo obstétrico en nuestra población local y colombiana, se hace necesario plantear esta investigación para luego evaluar su papel como ayuda diagnóstica.

Nuestro objetivo consiste en identificar la prevalencia de alteración del DUA y sus parámetros (IP y IR y persistencia del Notch) en pacientes de bajo riesgo obstétrico entre la semana 20 - 24,6 de gestación en Bucaramanga y su área metropolitana.

\section{MATERIALES Y MÉTODOS}

Este es un estudio transversal en el cual se incluyeron gestantes entre 18 - 35 años de edad, con máximo 24 6/7 semanas de edad gestacional que eran clasificadas en el control prenatal como pacientes de bajo riesgo obstétrico. El tamaño de muestra fue definido inicialmente en 207 pacientes teniendo en cuenta que en reportes anteriores se había encontrado prevalencia de alteración del DUA entre $6-42 \%$, la prevalencia de alteración del IP era del 17\%, este último era el parámetro que mostraba menor variabilidad y mayor relación con resultados perinatales adversos y desarrollo de preeclampsia por tal motivo fue elegido para definir el tamaño de muestra $(3,8,9)$. Debido que las poblaciones evaluadas tenían criterios de 
inclusión menos estrictos para pacientes de bajo riesgo obstétrico a los propuestos en el actual protocolo e incluían pacientes que podrían ser clasificados según nuestros parámetros como gestantes de alto riesgo, se consideró proponer una prevalencia menor al 15\%.

El reclutamiento se realizó entre enero y diciembre de 2017 en los primeros niveles de atención donde la Universidad Industrial de Santander hace presencia con rotaciones de Ginecología y Obstetricia del pregrado de medicina (Clínica Guane, Hospital San Juan de Dios de Girón), posteriormente se adicionó población que acudía al hospital de Piedecuesta con la finalidad de tener una muestra más representativa de la población. Se revisaron las historias clínicas de las pacientes y se aplicaron 2 encuestas (anexo 1 y 2) para corroborar que se tratara de pacientes de bajo riesgo obstétrico, la captación se realizó sin aleatorización según iban consultando las pacientes; posteriormente se citaron entre las 20 - 24 6/7 semanas de gestación para la realización de biometría fetal y toma del Doppler de arterias uterinas con ecógrafos GE Vóluson E6 y E8 y Medison Accuvix V10. La toma del DUA fue realizada por especialistas en Medicina Materno Fetal, con la finalidad de disminuir los errores de medición se estandarizó su toma con las guias Clinic de Barcelona publicadas en el 2014 (10); debido a que en la práctica clínica no se toman medidas para disminuir el error inter e intra - observador se asumió que la experticia de los especialistas era idónea. Solo se tuvieron en cuenta los datos de los reportes que describieran el peso fetal y el percentil de crecimiento, el IR e IP bilateral y las imágenes que permitieran evaluar la presencia del Notch de forma bilateral; aquellas pacientes que tuvieran reportes incompletos fueron excluidas de análisis estadistico. Los datos sociodemográficos y los valores reportados por los ecógrafos fueron consignados en el anexo 3, posteriormente fueron digitados en Excel y para el análisis estadístico se utilizó el programa Stata 12,1. A partir de los datos obtenidos se realizó una descripción de la población estudiada, tanto en sus variables cuantitativas, como cualitativas por medio de proporciones y medidas de tendencia central y dispersión.

La definición de alteración del IP y el IR se basó en el valor promedio bilateral con percentil mayor al 95 según las tablas reportadas en Ultrasonography in Obstetrics and Gynecology, vol 1. Stuttgart, New York, Thieme, 2005, page 469 - 480, 614 (8). 


\section{RESULTADOS}

Se analizaron los datos de 120 pacientes que fueron reclutadas entre el $1^{\circ}$ de enero y el 31 de diciembre de 2017. 65,8\% (79) de las pacientes residían en Bucaramanga y el restante pertenecían a su área metropolitana (Figura 1). El 53,0\% (63) pertenecían a estratos socioeconómicos 1 y 2 y el 47,0\% (57) eran de estratos socioeconómicos medios (Figura 2).

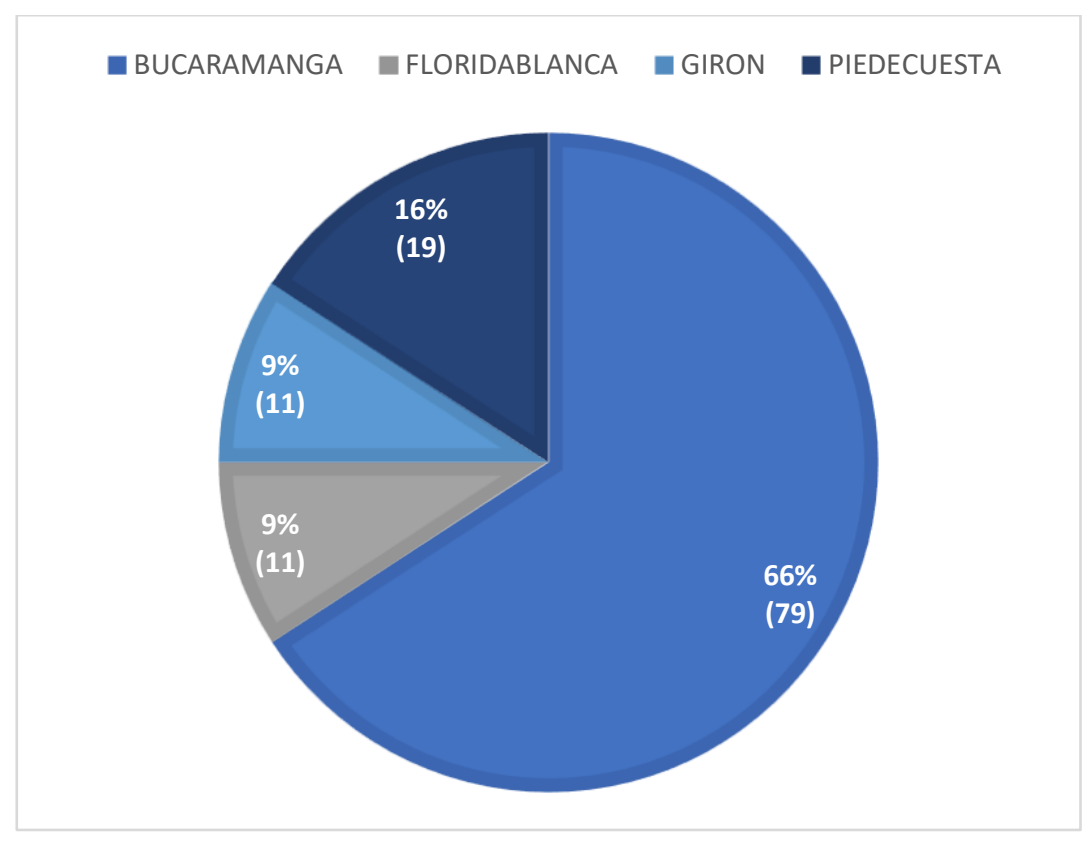

Figura 1. Municipio de procedencia de las pacientes de bajo riesgo obstétrico incluidas en la valoración del Doppler de Arterias Uterinas entre semana 20 -24,6. AMB 2017 


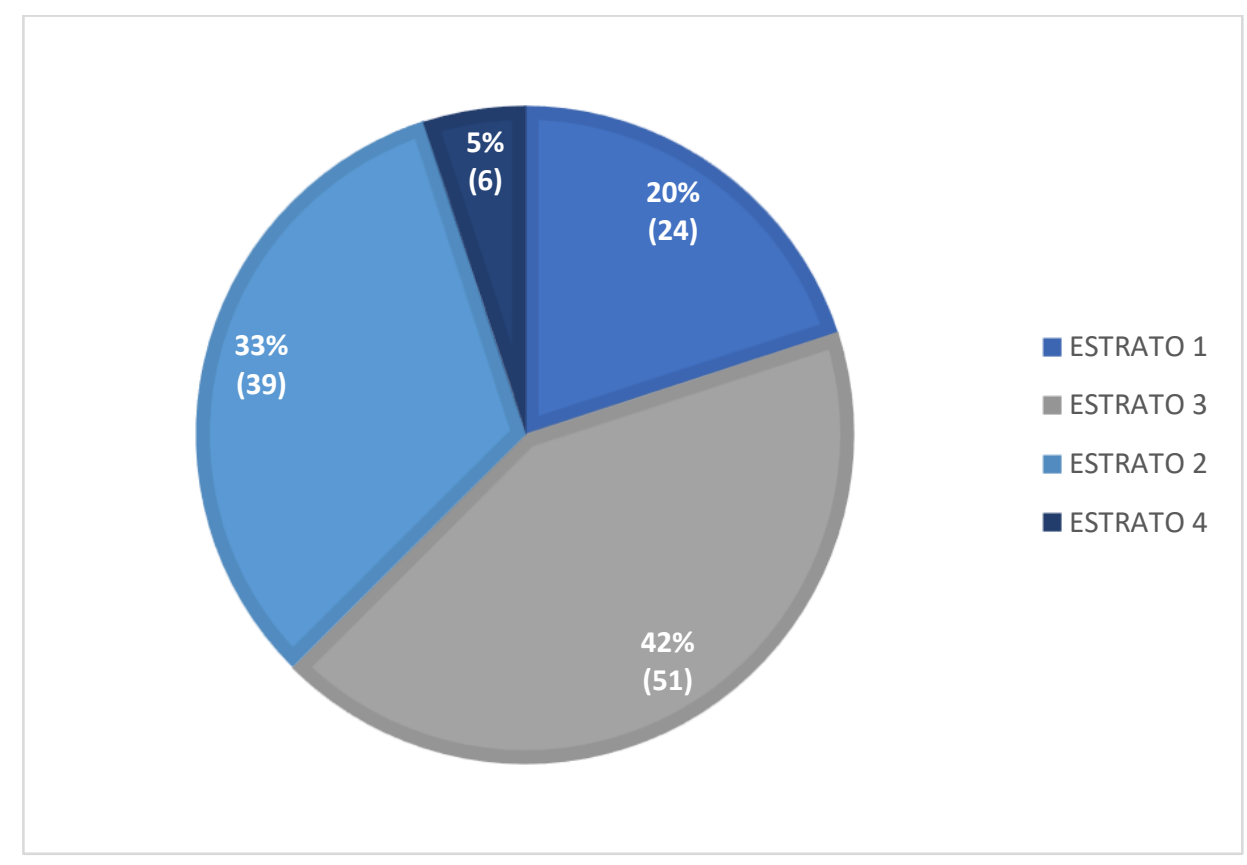

Figura 2. Estratificación Socioeconomica de las pacientes de bajo riesgo obstétrico incluidas en la valoración del Doppler de Arterias Uterinas entre semana 20 - 24,6. AMB 2017

Las pacientes tenían edades entre los 18 y los 35 años, la mediana de edad fue de 25 años (Recorrido Intercuartil (RIQ) de 21 - 30) (Figura 3); el IMC al inicio del embarazo tuvo una mediana de 23,03 (RIQ de 21,34 - 25,65). Las pacientes se encontraban entre su $1^{\circ}$ y $5^{\circ}$ embarazo, 2 estaban en su $5^{\text {a }}$ gestación y 59 (49,17\%) eran pacientes primigestantes (Figura 4). Del total de las pacientes evaluadas, 2 (1,67\%) tenían antecedente familiar de trastornos hipertensivo en el embarazo en 1er grado de consanguinidad y ambas presentaron Doppler de arterias uterinas reportados como normales. 


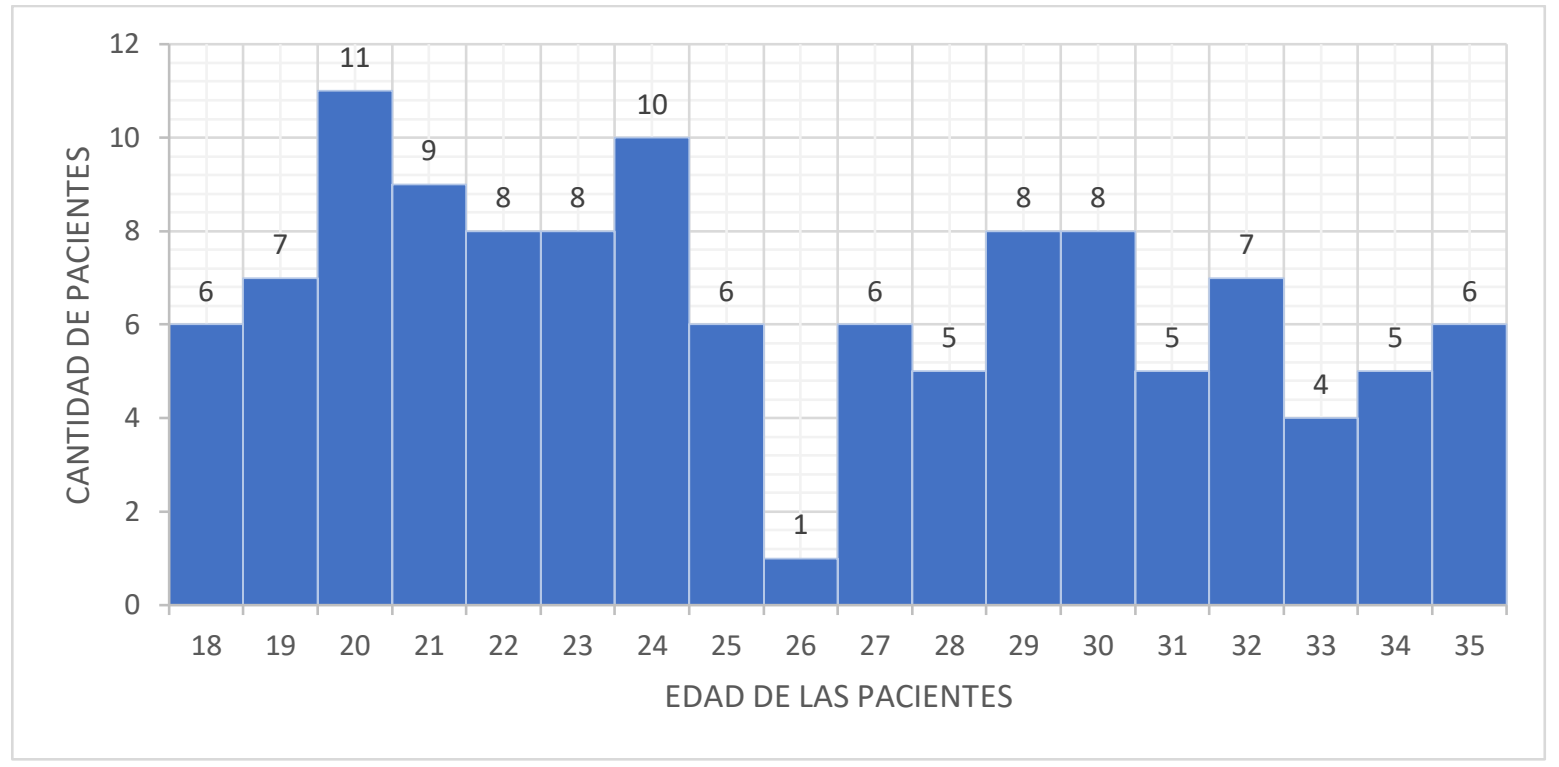

Figura 3. Edad de las pacientes de bajo riesgo incluidas en valoración del Doppler de Arterias Uterinas entre semana $20-24,6$. AMB 2017

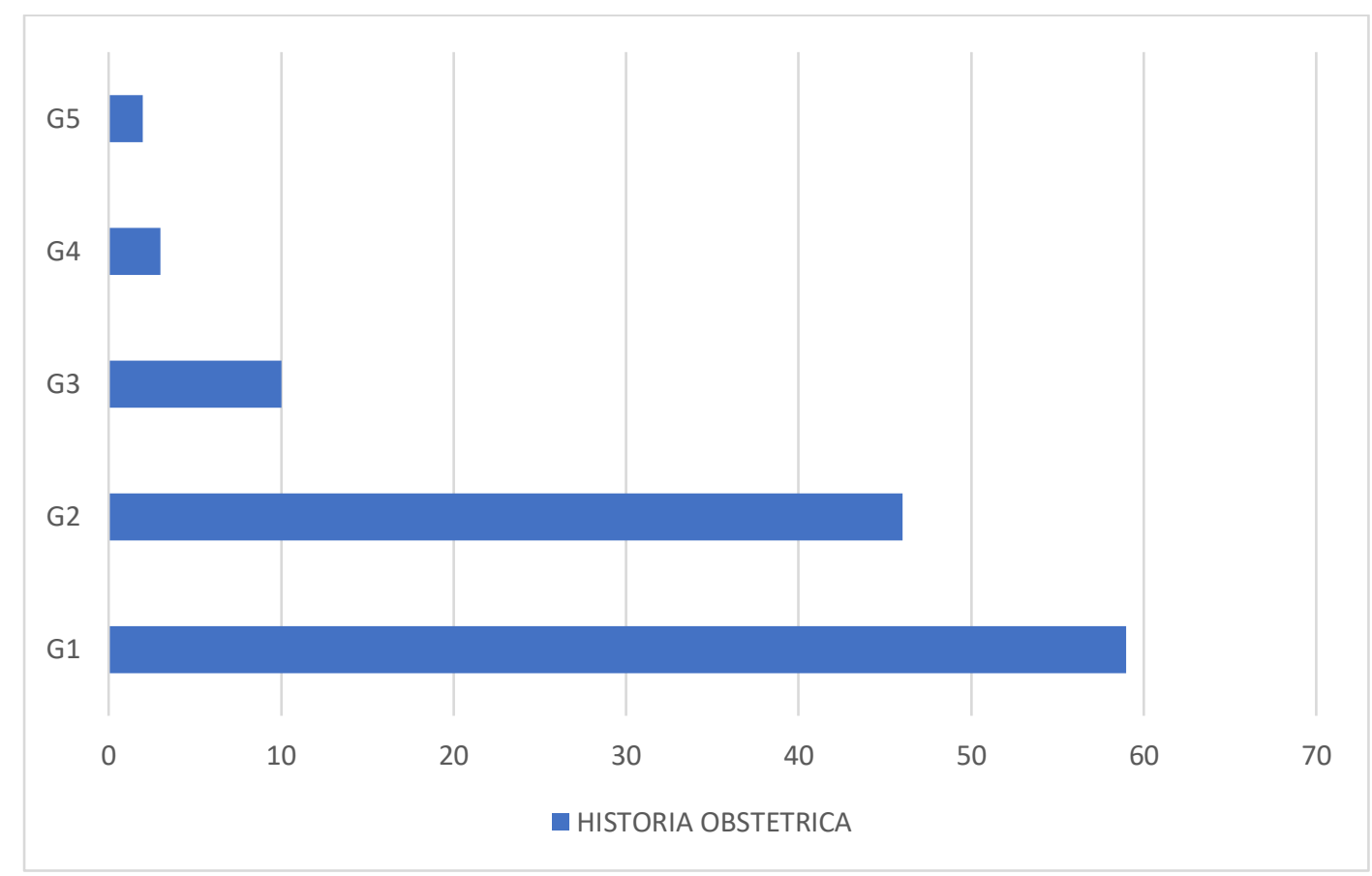

Figura 4. Gestaciones de las pacientes estudiadas incluyendo actual embarazo que son pertenecientes al AMB durante el 2017

$\mathrm{G}=$ No. De gestaciones, sin importar el desenlace final de estas.

En la valoración fetal se observó que la media en la edad gestacional al momento de realizar el Doppler de arterias uterinas fue de 21.6 semanas, el 52,5\% (63) tenían entre 20.0 y 21,6 semanas, el $30,0 \%$ (36\%) estaban entre las 22,0 y las 23,6 semanas y $17,5 \%$ (21) restante 
tenían entre 24,0 y 24,6 semanas; el crecimiento de ellos se encontró entre el percentil 10 y 95, la mediana se presentó en el percentil 53,6 (RIQ 35 - 74,7), como hallazgo incidental se encontró 1 feto creciendo en el percentil 4, al reevaluar los factores de riesgo en la historia obstétrica no se encontraron criterios de exclusión (Figura 5).

Se encontró anormalidad del IP en el 15,0\% (18) de las pacientes con un IC 95\% de 9.1\% a 22.7\%, del IR en el 25,0\% (30) de las mismas con un IC $95 \%$ de $17.3 \%$ a $33.7 \%$ y presencia del Notch en el 10,8\%, (13) de ellas con IC 95\% de 5.9\% a 17.8\% (Figura 6).

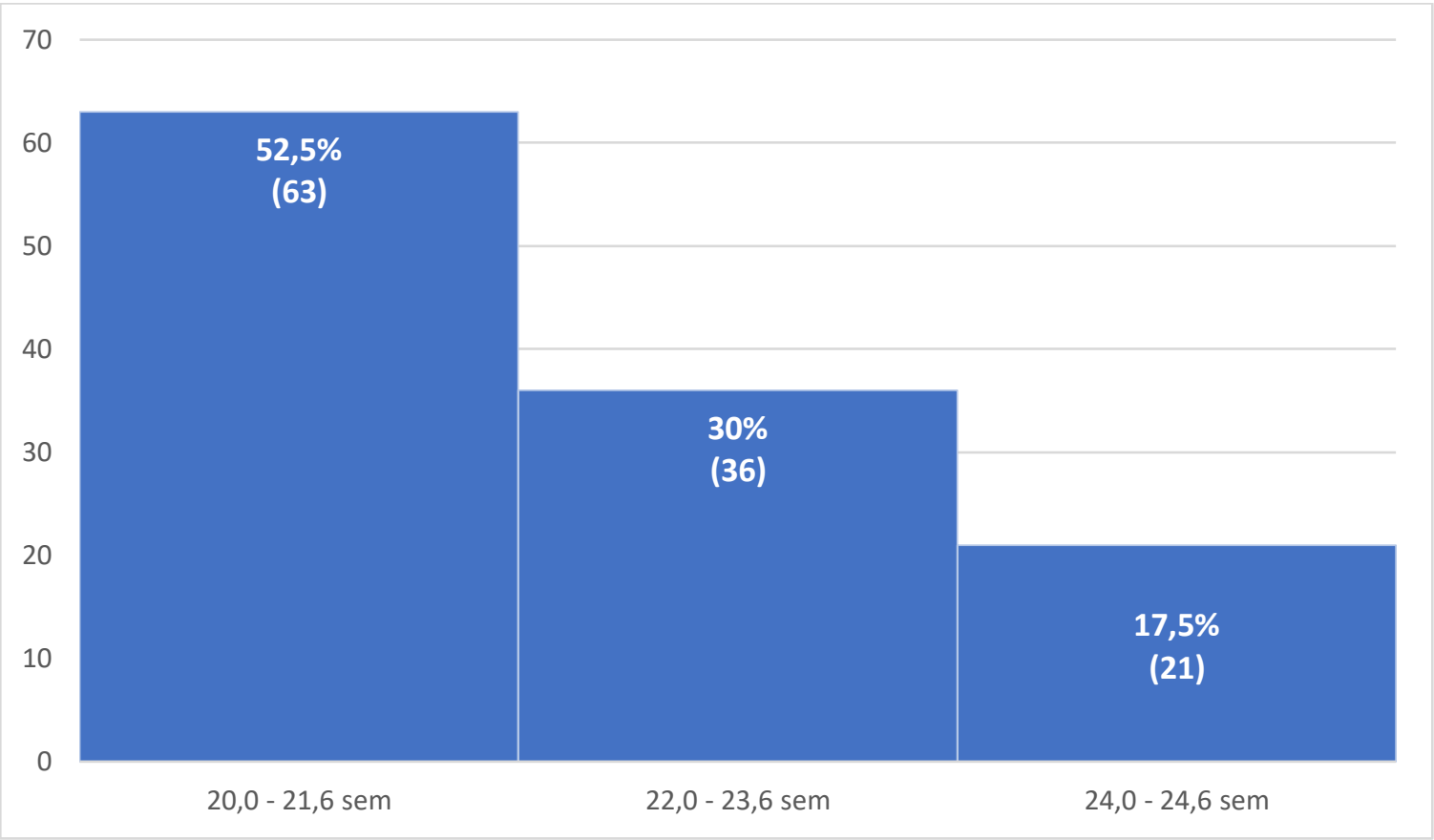

Figura 5. Edad gestacional a la cual se realizó el Doppler de Arterias Uterinas, en las pacientes evaluadas pertenecientes al AMB durante el año 2017 


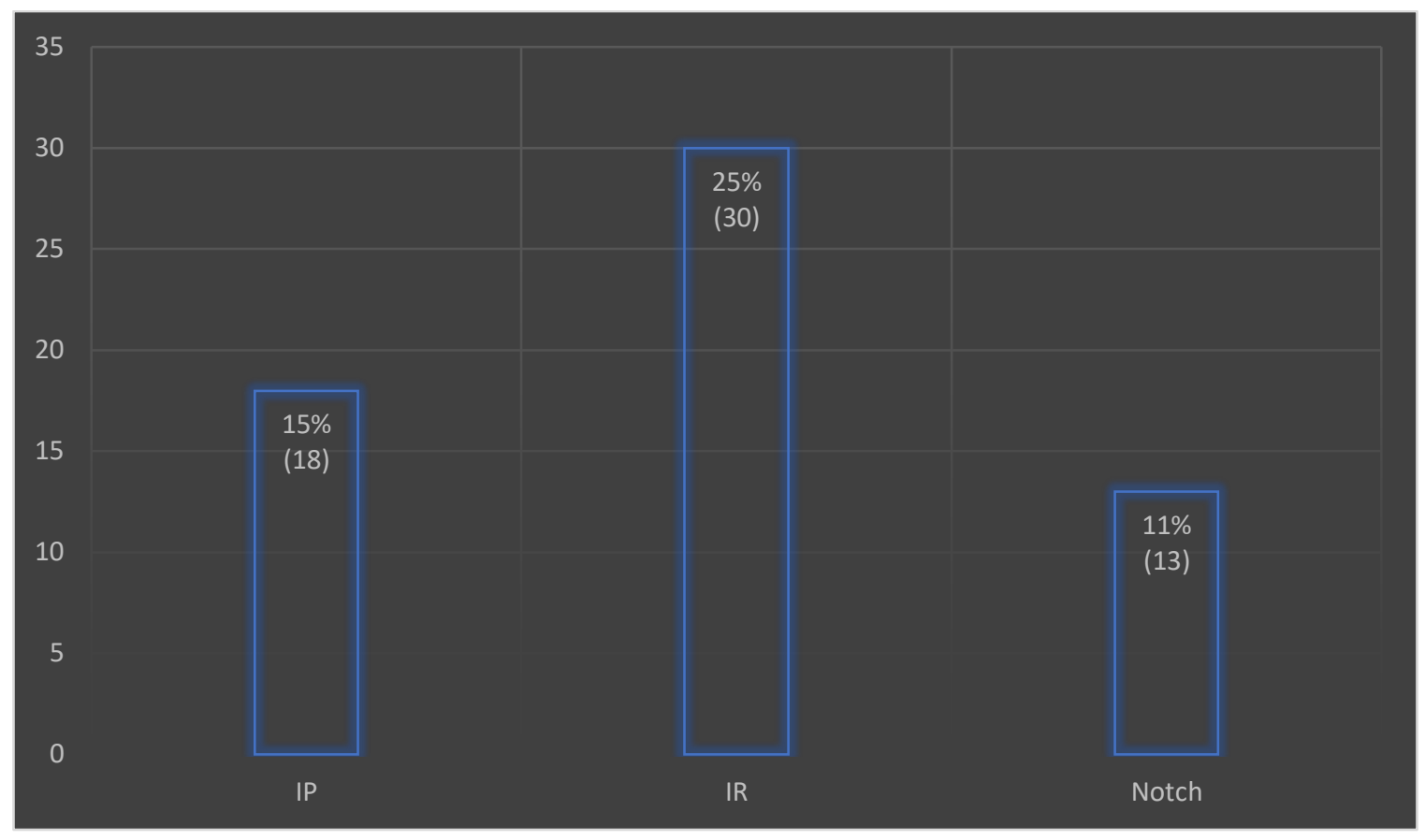

Figura 6. Frecuencia de alteración de cada uno de los parámetros evaluados en el Doppler de Arterias uterinas en pacientes de bajo riesgo entre semana $20-24,6$.

Dieciocho (15,0\%) pacientes que tenían IP alterado 2 tenían IR normal y en la valoración subjetiva del Notch este se encontraba ausente en 6 (Tablas 1 y 2).

De los 3 parámetros evaluados, se observó que el parámetro que se presentaba alterado con mayor frecuencia fue el IR.

TABLA 1. RELACIÓN ENTRE LA ALTERACIÓN DEL IP Y EL IR

\begin{tabular}{|c|c|c|c|}
\hline \multirow{2}{*}{ IR } & \multicolumn{2}{|c|}{ IP } & TOTAL \\
\cline { 2 - 4 } & NORMAL & ANORMAL & \\
\hline NORMAL & 88 & 2 & 90 \\
\hline ANORMAL & $86,27 \%$ & $11,11 \%$ & $75 \%$ \\
\hline TOTAL & 14 & 16 & 30 \\
\hline & $13,73 \%$ & $88,89 \%$ & 120 \\
\hline
\end{tabular}


TABLA 2. RELACIÓN ENTRE LA ALTERACIÓN DEL IP Y EL NOTCH
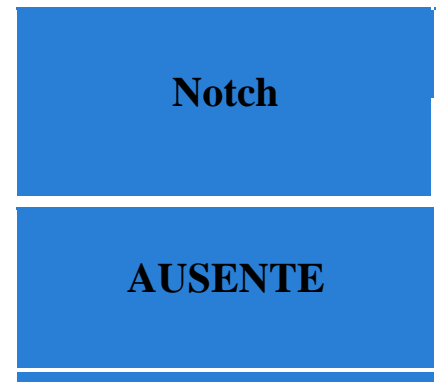

PRESENTE

TOTAL

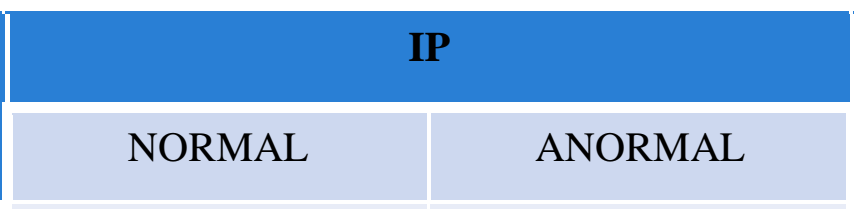

101
6

$33,33 \%$

12

$66,67 \%$

18

$100 \%$

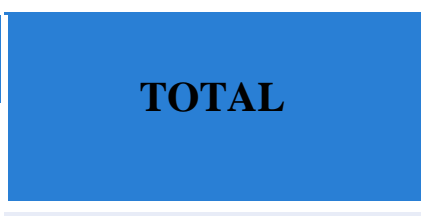

107

$89,17 \%$

13

$10,83 \%$

120

$100 \%$

Al considerar la interpretación de los valores según las tablas estandarizadas de los equipos utilizados, fueron considerados como estudios normales según el IP el 87,50\% (105) de los reportes y anormales el 12,50\% (15). Los 3 Doppler de arterias uterinas que tenían diferencias en el reporte tenían los siguientes valores: IP 1,41 a las 20,5 semanas, 1,34 a las 21,1 semanas y 1,45 a las 21,2 semanas.

\section{DISCUSIÓN}

El presente estudio se planteó con la finalidad de establecer si hay cambios en la prevalencia de las alteraciones del Doppler de arterias uterinas en el segundo trimestre en pacientes categorizadas como bajo riesgo debido a que la población estudiada tiene diferencias sociodemográficas y de clasificación con respecto a los estudios previamente realizados $(3,4,9)$.

La mediana de edad en la población a estudio fue de 25 años con RIQ 21 - 30 años; según el reporte preliminar del DANE para el año 2017 el 55,13\% (6223) de las pacientes tenían 20 29 años, haciendo evidente que la muestra era representativa de nuestra población en cuanto 
al tópico de edad que era uno de los puntos identificados con disparidad frente a los estudio de Jamal y col, Becker y Col y Schwarze y col $(3,4,9,11)$.

Jamal y colaboradores encontraron que la prevalencia de alteración del índice de pulsatilidad era del 17,2\% (N=23), sin embargo, la población a estudio fue definida como bajo riesgo por ser gestaciones únicas con ausencia del uso de medicamentos como el ácido acetil salicílico o heparinas y por la ausencia de malformaciones fetales, no incluyeron los antecedentes obstétricos como criterios de exclusión; en un estudio publicado por el grupo de Maciel la prevalencia del IP en $\mathrm{P}>95$ fue del 6,2\% $(\mathrm{N}=10)$, esta era una población con características similares a la nuestra como edad y paridad y tuvieron criterios más estrictos para la inclusión y exclusión de pacientes $(9,12)$. Por lo tanto, el hallazgo en la prevalencia del IP en P >95 del 15\% (N=18) (IC 95\% de 9.1-22.7) se correlacionaba con la hipótesis inicialmente planteada que debía ser menor si se afinaban los criterios de selección, pero podía ser variable dependiendo de la población estudiada. Goffinet F. y colaboradores también describió en su estudio una prevalencia de alteraciones del Doppler de arterias uterinas en pacientes de bajo riesgo a partir del Notch del 10,5\% que al compararlo con la prevalencia en la aparición del Notch en el presente estudio $(10,8 \%(\mathrm{~N}=13)$ IC $95 \%(5.9 \%$ a 17.8)) eran similares (13). No se realizó un análisis más detallado con respecto al IR por evidenciar en la literatura que el parámetro con mejor correlación entre los desenlaces adversos y su alteración fue el IP (9).

Es importante recalcar que hubo diferencias en la definición de alteración o normalidad en el Doppler de arterias uterinas al comparar los datos tomando las tablas estandarizadas que trae los ecógrafos utilizados en la investigación y los datos de las tablas del texto de referencia, hecho que hizo cuestionarnos sobre cuáles son los instrumentos más precisos y válidos a utilizar en la evaluación de nuestra población y proponemos un estudio posterior que evalúe la reproducibilidad de estos instrumentos de medición como lo había publicado Figueira en su estudio donde encontró diferencias en los valores absolutos del P95 del IP del Doppler de arterias uterinas en una población Brasilera entre las 16 - 41 semanas (14).

Como fortalezas del estudio presentamos el proceso de recolección de las pacientes que se realizó a partir de la identificación de las pacientes de bajo riesgo por historia clínica, datos 
del carnet del CPN e interrogatorio dirigido (Anexo 1 y 2), excluyendo las pacientes que tuvieran factores de riesgo para desenlaces adversos que estuvieran correlacionados con la placentación a diferencia de los estudios presentados por Becker y Jamal (5, 20). Otra fortaleza importante fue la realización de los exámenes por el equipo de especialistas en Medicina Materno Fetal de la Unidad de Medicina Materno Fetal (UMMF) HUS - UIS considerados personal altamente entrenado en dichas evaluaciones ecográficas.

Dentro de las debilidades se reporta el hecho de no haber logrado el tamaño de muestra inicialmente propuesto (207 pacientes) y la ausencia de pacientes de estratos 5 y 6 , sin embargo, se recalca que con el tamaño de muestra se encontró la prevalencia propuesta.

Este es un estudio valioso al evidenciar que las pacientes clasificadas como bajo riesgo obstétrico en Bucaramanga y su área metropolitana presentan una proporción de alteración del Doppler de arterias uterinas no despreciable y conociendo la cantidad de nacimientos/año en la región podría ser utilizada como argumento para justificar una nueva investigación que evalué la asociación con eventos adversos $(22,34)$; adicionalmente es el primer estudio en población colombiana de bajo riesgo obstétrico evaluadas en el segundo trimestre que reporta la prevalencia de alteración del Doppler de arterias uterinas según los tres items evaluados en la literatura (IP, IR y notch). Recalcamos que es el estudio base para establecer una línea de investigación de la UMMF y su grupo GINO en la predicción de resultados perinatales adversos dado que en la literatura se ha establecido la asociación entre la alteración del Doppler de arterias uterinas en segundo trimestre y la presencia de desenlaces desfavorables de la gestación como: RCIU, muerte intrauterina, preeclampsia y abrutio placentae $(3,5,12,15,16)$.

Por lo tanto, se recomienda continuar evaluando la correlación de la alteración del Doppler de arterias uterinas y las patologías derivadas de la alteración en la placentación. Posteriormente definir la pertinencia de incorporar este examen como parte del tamizaje del control prenatal de la población de bajo riesgo de Bucaramanga y su área metropolitana para reclasificar las pacientes con alteración del Doppler de uterinas como gestantes de alto riesgo 
obstétrico, las cuales deben ser evaluadas y manejadas adecuadamente por los grupos de Ginecoobstetricia y Perinatologia.

Teniendo en cuenta que no fue posible lograr la muestra inicialmente propuesta y que no se realizó la selección de las pacientes de forma aleatoria consideramos que la muestra no es representativa de la población, sin embargo, los hallazgos son aplicables para definir el tamaño de muestra en los estudios relacionados con Doppler de arterias uterinas en segundo trimestre en pacientes de bajo riesgo obstétrico que sean realizados en el territorio colombiano.

\section{FINANCIACIÓN}

La financiación proviene de la Universidad Industrial de Santander al ser la institución académica a la que pertenecen como docentes de catedra los especialistas en medicina materno fetal que participaron en la toma del DUA; ninguno de ellos participó en el análisis estadístico de los datos. Como autora principal financié los gastos suplementarios requeridos para el reclutamiento de las pacientes teniendo en cuenta que el actual estudio fue planteado como mi tesis de grado la cual fue aprobada el 26 de enero del presente año.

\section{BIBLIOGRAFÍA}

1. Ferri F. Ferri's clinical advisor 2016. Elsevier 2016:1004-5.

2. Herrera $\mathrm{J}$, et al. Evaluación periódica del riesgo biopsicosocial prenatal en la predicción de las complicaciones maternas y perinatales en Asia 2002-2003. Colomb Med. 2006;(3) supl 1:6-14.

3. Schwarze A, et al. Doppler ultrasound of the uterine artery in the prediction of severe complications during low risk pregnancies. Arch gynecol Obstet. 2005;(271):46-52.

4. Becker R, Vonk R. Doppler Sonography of Uterine arteries at 20-23 weeks: depth of notch gives information on probability of adverse pregnancy outcome and degree of fetal growth restriction in a low risk population. Fetal Diagn Ther. 2010;(27):7886.

5. Harrington K, Cooper D, Lees C, Hecher K, Campbell S. Doppler ultrasound of the uterine arteries: the importance of bilateral notching in the prediction of preeclampsia, 
placenta abruption or delivery of a small for gestational age baby. Ultrasound Obstet Gynecol. 1996;(7):182-188.

6. Sepúlveda E, Crispi F, Pons A, Gratacos E. Restricción de crecimiento intrauterino, Rev Med Clin Condes. 2014;25(6):958-63.

7. Peña H, Camacho M, Escobedo F. Velocimetría Doppler de las arterias uterinas en el embarazo. Rev Esp Med Quir. 2008;13(4):177-180.

8. Callen PW. Ultrasonography in obstetrics and gynecology. 5th ed. Philadelphia. Saunders Elsevier; 2008. p. 794-807.

9. Jamal A, Abbasalizadeh F, Vafaei H, Marsoosi V, Eslamian L. Multicenter screening for adverse pregnancy outcomes by uterine artery Doppler in the second and third trimester of pregnancy. Med Ultrason. 2013;15(2):95-100

10. Figueras F, Puerto B. Guia clínica: Doppler en medicina fetal. Servicio de medicina fetal. [Internet]. Barcelona, España: 2014. Disponible en: https://medicinafetalbarcelona.org/clinica/images/protocolos/patologia_fetal/doppl er.pdf.

11. DANE Colombia. Nacimientos por grupos de edad de la madre, según el departamento y municipio de residencia de la madre. [Internet]. Colombia: 2017. Disponible en: https://www.dane.gov.co/index.php/estadisticas-portema/salud/nacimientos-y-defunciones/nacimientos/nacimientos-2017.

12. Scandiuzzi RM, et al. Maternal uterine artery Doppler in the first and second trimesters as screening method for hypertensive disorders and adverse perinatal outcomes in low-risk pregnancies. Obstet Gynecol Sci. 2016;59(5):347-356.

13. Goffinet F,et al. Screening with a uterine Doppler in low risk pregnant women followed by low dose aspirin in women with abnormal results: a multicenter randomised controlled trial. Br J Obstet Gynaecol. 2001;(108):510-518.

14. Figueira C, et al. Fetal Hemodynamic Parameters in Low Risk Pregnancies: Doppler Velocimetry of Uterine, Umbilical, and Middle Cerebral Artery. Scientific World Journal [Internet]. 2016. Disponible en: http://dx.doi.org/10.1155/2016/1693704.

15. Tayyar A, Guerra L, Wright A, Wright D, Nicolaides KH. Uterine artery pulsatility index in the three trimesters of pregnancy: effects of maternal characteristics and medical history. Ultrasound Obstet Gynecol. 2015;(45):689-97. 
16. Afrakhteh M, Moeini A, Sanei M, Haghighatkhah H, Fakhri M, Masoom N. Uterine Doppler velocimetry of the uterine arteries in the second and third trimesters for the prediction of gestational outcome. Rev Bras Ginecol Obstet. 2014;36(1):35-9.

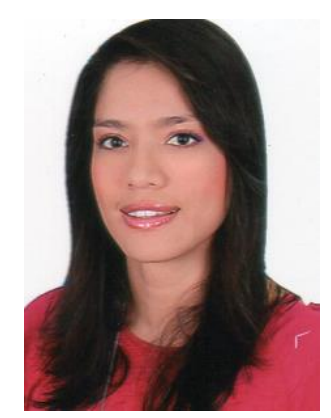

LINDA PATRICIA MELÉNDEZ PÉREZ is a specialist in Gynecology and Obstetrics from Colombia. She studied medicine at the Universidad Industrial de Santander - UIS in Colombia where she also became a gynecologist and obstetrician. In her third year of specialization she was chief resident and participated in the coordination of activities in the academic program. 


\section{Anexo 1. \\ Formato de verificación de los criterios de inclusión}

Fecha:

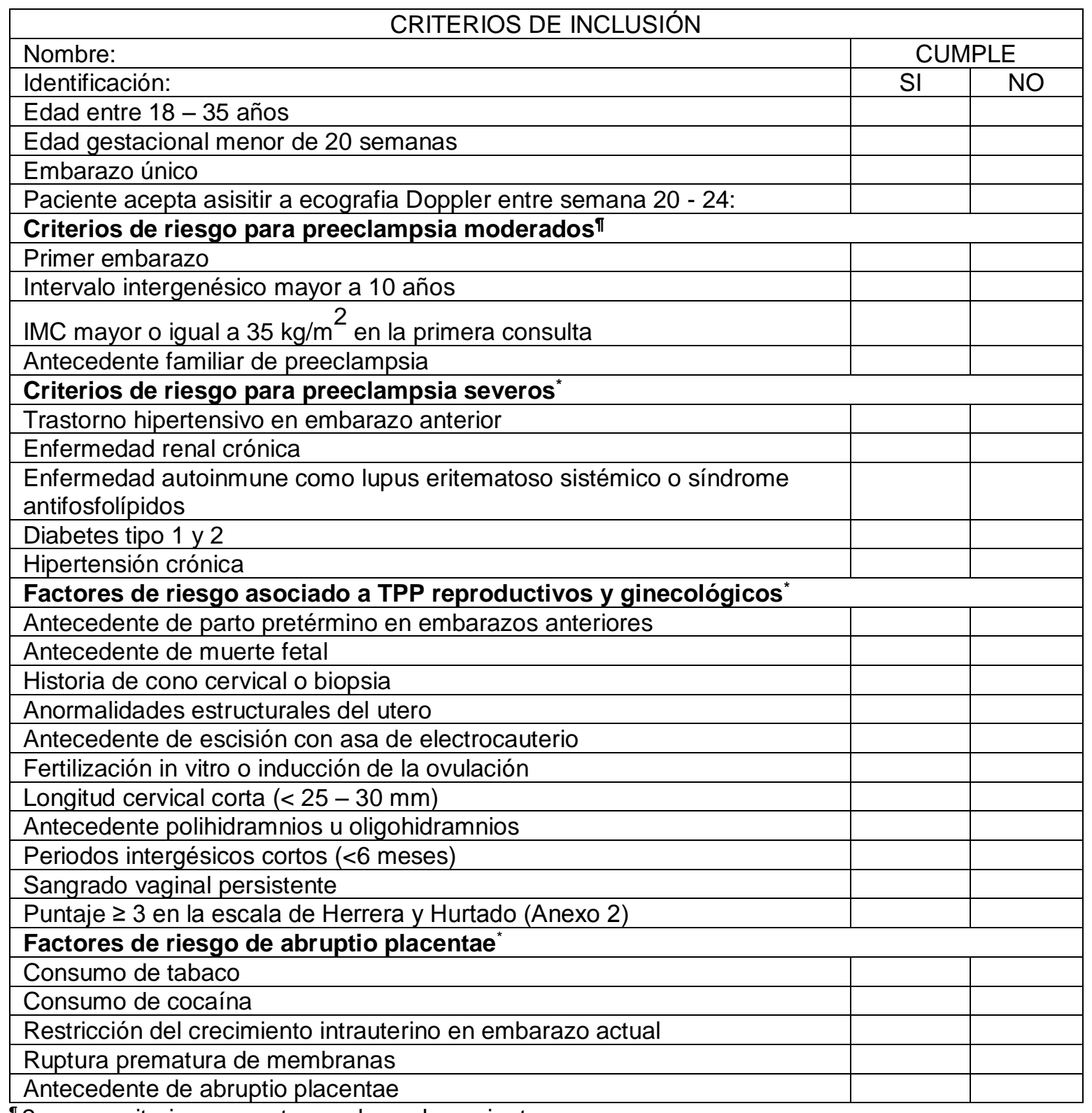

ॠ 2 o mas criterios presentes excluyen la paciente

${ }^{*} 1$ criterio presente excluye la paciente 
Anexo 2.

Escala de Herrera y Hurtado para la evaluacion del riesgo Psicosocial

\begin{tabular}{lcc}
\hline CARACTERÍSTICA & CLASIFICACIÓN & PUNTOS \\
\hline HISTORIA REPRODUCTIVA & & \\
\hline Edad & $16-35$ años & 1 \\
& $>35$ años & 0 \\
Paridad & 0 & 2 \\
& $1-4$ & 1 \\
Cesárea previa & $>4$ & 0 \\
Preeclampsia o hipertensión & & 1 \\
Abortos recurrentes o infertilidad & & 1 \\
Hemorragia post parto o remoción manual de la & & 1 \\
placenta & gr & 1 \\
Peso del recién nacido & & 1 \\
Mortalidad fetal tardía o neonatal temprana & & 1 \\
Trabajo de parto anormal o dificultoso & & 1 \\
Cirugía ginecológica previa & & 1 \\
\hline
\end{tabular}

\section{EMBARAZO ACTUAL}

Enfermedad renal crónica

Diabetes gestacional

Diabetes preconcepcional

Hemorragia

Anemia

Embarazo prolongado

Hipertensión arterial

2
2
3

Polihidramnios

$<20$ semanas

$\geq 20$ semanas

$\mathrm{Hb}<10 \mathrm{~g} / \mathrm{dl}$

$>42$ semanas

Embarazo múltiple

Presentación de frente o transverso

${ }^{*}$ Ansiedad severa: Tensión emocional, humor depresivo, síntomas neurovegetativos de angustia (dos o tres intensos)

^Soporte social inadecuado: Insatisfacción con el apoyo recibido por la familia y el compañero en tiempo, espacio y dinero (dos o tres aspectos insatisfechos) 
Anexo 3.

Formato para el registro de variables.

\section{PREVALENCIA DE LA ALTERACIÓN DEL DOPPLER DE ARTERIAS UTERINAS ENTRE SEMANA 20 - 24 EN PACIENTES DE BAJO RIESGO}

Institucion:

Nombre: Código:

Edad: Documento:

Fecha de nacimiento:

Dirección domicilio:

Teléfono:

Celular:

Nombre de familiar: Celular familiar:

Formula obstétrica:

Antecedente familiar de preeclampsia:

Peso: Talla: IMC:

\section{RESULTADOS}

Resultado de Doppler de arterias uterinas: Positivo Negativo

Edad gestacional de toma de Doppler:

Peso fetal estimado en ecografía obstétrica:

Percentil:

IP derecho:

IR derecho: IP izquierdo: IR izquierdo: IP medio: Percentil:

Notch presente: Notch ausente: IR medio: Percentil:

\section{CRITERIOS DE EXCLUSIÓN}

Retiro de consentimiento informado:

Diagnóstico de embarazo multiple:

Diágnostico de malformaciones fetales mayores:

Otra alteración que implique alto riesgo para la gestación: 